\title{
Drusen deposits associated with aging and age-related macular degeneration contain nonfibrillar amyloid oligomers
}

\author{
Volker Luibl, ${ }^{1}$ Jose M. Isas, ${ }^{1}$ Rakez Kayed, ${ }^{2}$ Charles G. Glabe, ${ }^{2}$ Ralf Langen, ${ }^{1,3}$ and Jeannie Chen ${ }^{1,4}$ \\ ${ }^{1}$ Beckman Macular Research Center, Doheny Eye Institute and Zilkha Neurogenetic Institute, University of Southern California Keck School of Medicine, \\ Los Angeles, California, USA. '2Department of Molecular Biology and Biochemistry, University of California, Irvine, California, USA. \\ ${ }^{3}$ Department of Biochemistry and Molecular Biology and ${ }^{4}$ Departments of Cell and Neurobiology and Ophthalmology, \\ University of Southern California Keck School of Medicine, Los Angeles, California, USA.
}

\begin{abstract}
Protein misfolding and aggregation are thought to underlie the pathogenesis of many amyloid diseases, such as Alzheimer and Parkinson diseases, whereby a stepwise protein misfolding process begins with the conversion of soluble protein monomers to prefibrillar oligomers and progresses to the formation of insoluble amyloid fibrils. Drusen are extracellular deposits found in aging eyes and in eyes afflicted with age-related macular degeneration (AMD). Recent characterizations of drusen have revealed protein components that are shared with amyloid deposits. However, characteristic amyloid fibrils have thus far not been identified in drusen. In this study, we tested the hypothesis that nonfibrillar oligomers may be a common link in amyloid diseases. Oligomers consisting of distinct amyloidogenic proteins and peptides can be detected by a recently developed antibody that is thought to recognize a common structure. Notably, oligomers exhibit cellular toxicity, which suggests that they play a role in the pathogenesis of neurodegenerative diseases. Through use of the antioligomer antibody, we came to observe the presence of nonfibrillar, toxic oligomers in drusen. Conversely, no reactivity was observed in age-matched control eyes without drusen. These results suggest that amyloid oligomers may be involved in drusen biogenesis and that similar protein misfolding processes may occur in AMD and amyloid diseases.
\end{abstract}

\section{Introduction}

The formation of insoluble extracellular deposits consisting of misfolded, aggregated protein is a hallmark of many neurodegenerative diseases. Extracellular deposits are also present in aging human eyes and in eyes afflicted by age-related macular degeneration (AMD). These deposits, called drusen, are found beneath the basement membrane of the retinal pigmented epithelium (RPE) and the inner collagenous layer of the Bruch membrane $(\mathrm{Bm})$. Despite the well-established correlation between the presence of drusen and AMD, the underlying cause of drusen formation and its role in RPE and photoreceptor cell degeneration are not fully understood (1-3).

Recent evidence suggests that drusen formation and AMD share some similarities with amyloid diseases such as Alzheimer disease (AD) and Parkinson disease (PD). Like AMD, amyloid diseases are strongly correlated with advancing age and the formation of deposits. Moreover, these amyloid deposits contain a wide range of lipids and proteins, many of which are also present in drusen. Shared components of amyloid deposits and drusen include proteins such as vitronectin, amyloid $\mathrm{P}$, apolipoprotein $\mathrm{E}$, and even the amyloid $\beta(\mathrm{A} \beta)$ peptide that is associated with amyloid plaques in $\mathrm{AD}(4-6)$. In humans, the $A P O E^{*} 4$ allele shows a strong positive association with $\mathrm{AD}$. Interestingly, expression of

Nonstandard abbreviations used: $A \beta$, amyloid $\beta ; A D$, Alzheimer disease; $A M D$, agerelated macular degeneration; $\mathrm{Bm}$, Bruch membrane; IAPP, islet amyloid polypeptide; MTT, 3-[4,5-dimethylthiazol-2-yl]-2,5-diphenyl tetrazolium bromide; PD, Parkinson disease; RPE, retinal pigmented epithelium.

Conflict of interest: The authors have declared that no conflict of interest exists. Citation for this article: J. Clin. Invest. 116:378-385 (2006). doi:10.1172/JCI25843. the $A P O E^{*} 4$ allele in transgenic mice leads to ocular changes that mimic the pathology associated with human AMD (7). In addition, acute phase reactants, complement components, immune modulators, and other inflammatory mediators are present in amyloid deposits as well as in drusen, suggesting a possible common role for the inflammatory pathway in AMD and amyloid diseases (8-10). It is particularly noteworthy that the presence of complement components such as C5, C5b9 and C3 fragments had been observed in drusen of varying sizes and shapes, from small, "hard" drusen to large, "soft" drusen, in aging eyes as well as in AMD eyes (9-11). These observations are consistent with the idea that complement activation may be involved in drusen biogenesis. Together with the recent discovery that a polymorphism in complement factor $\mathrm{H}$ increases the risk factor of AMD (12-15), substantial attention is now focused on the role of inflammation in the pathogenesis of this disease.

Despite the shared similarities mentioned above, AMD has thus far not been classified as an amyloid disease. Among the principal differences is the fact that classical amyloid diseases typically exhibit large amounts of amyloid fibrils (16). For example, in the case of $\mathrm{AD}$, the characteristic plaques consist primarily of fibrillar Alzheimer $A \beta$ peptide, while the Lewy bodies found in PD are abundant in $\alpha$-synuclein fibrils. These amyloid fibrils are elongated, 6- to 15-nm-wide rod-like structures of indeterminate length that are characterized by a common cross $\beta$ structure (17). In addition to their related structural features, amyloid fibrils display characteristic tinctorial properties, such as thioflavin $\mathrm{T}$ and congo red staining (18-20). Though drusen do stain with thioflavin $\mathrm{T}$ and congo red, the characteristic apple-green birefringence often seen in congo red-stained amyloid fibrils is not present (5, 

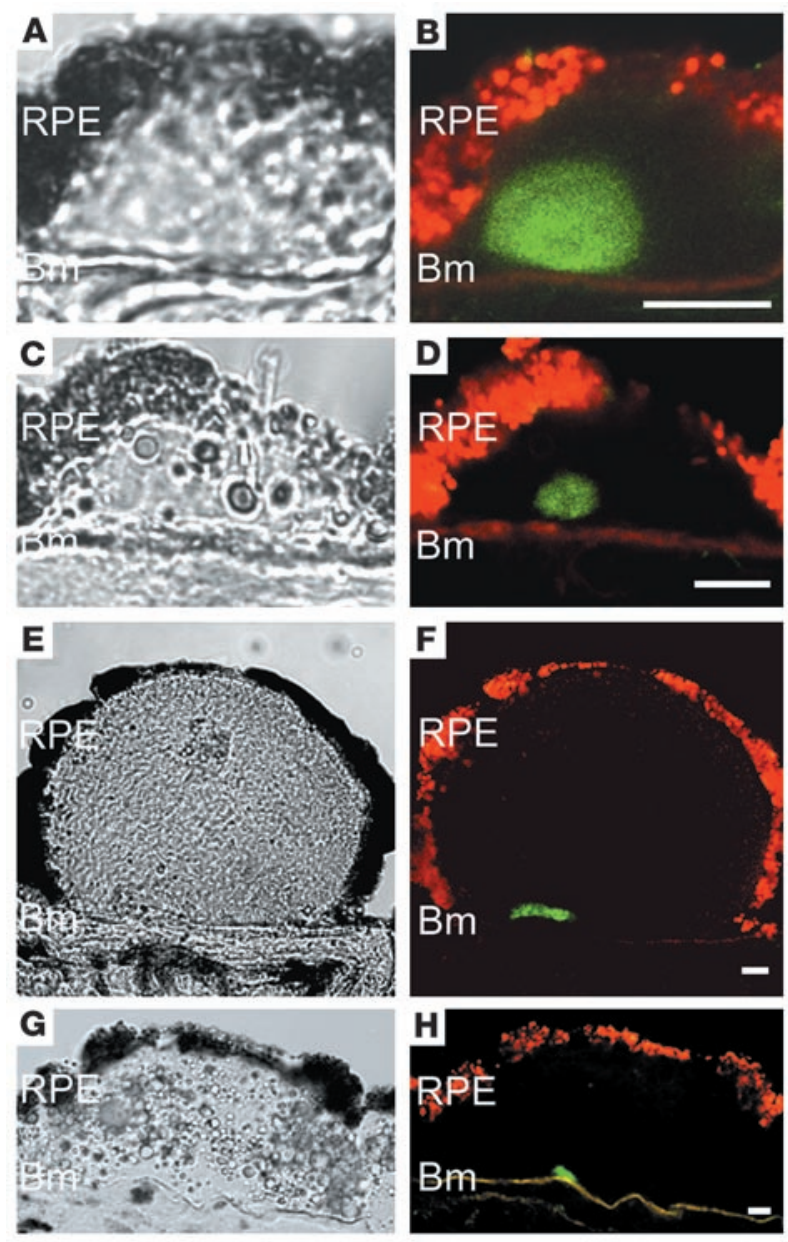

6). Although amyloid proteins such as the $\mathrm{A} \beta$ peptide, transthyretin, immunoglobulin light chains, and amyloid $A$ are found in drusen and sub-RPE deposits (4-6, 9, 21), electron microscopy studies have yielded sparse evidence of the presence of bona fide amyloid fibrils. These observations have precluded AMD from being viewed as a classical amyloid disease.

Amyloid fibril formation is a multistep protein misfolding cascade of molecular events, wherein a monomeric protein undergoes a conformational reorganization into a number of different oligomeric, $\beta$ sheet-containing structures that ultimately convert into amyloid fibrils $(22,23)$. Numerous studies of various amyloid diseases have led to the perception that nonfibrillar oligomers, rather than amyloid fibrils, might be the primary toxic agents (23-30). This notion has been supported by animal models demonstrating that amyloid fibrils do not seem to be required for the pathogenesis of amyloid diseases (30-34). These results suggest that additional amyloid diseases might be identified wherein pathogenic nonfibrillar oligomers are present without considerable accumulations of amyloid fibrils. Recent evidence suggests that desminrelated cardiomyopathy may be such a disease (35).

The central goal of the present study was to investigate whether nonfibrillar amyloid oligomers are present in drusen. To address this question, we took advantage of a recently developed A11 "anti-oligomer" antibody that specifically recognizes nonfibrillar oligomers (24). Although this antibody was initially raised against a molecular mimic of nonfibrillar oligomer made from the Alzheimer A $\beta$

\section{Figure 1}

Immunolocalization of amyloidogenic oligomers in drusen by confocal laser microscopy. (A, C, E, and G) Differential interference contrast images. (B, D, F, and $\mathbf{H}$ ) Confocal fluorescence images of amyloid oligomer cores (green, FITC channel). Drusen exhibit amyloid oligomer reactivity in the form of a core-like structure that accumulates centrally within drusen and in close proximity to the Bm. Autofluorescence of lipofuscin granules in the RPE cytoplasm is shown in red (Cy3 channel). (A and $\mathbf{B}$ ) Anti-oligomer-specific antibody recognizes a spherical structure $(\sim 15 \mu \mathrm{m})$ in a small druse $(\sim 30 \mu \mathrm{m})$. (C-F) Two larger drusen with centrally located core structure. ( $\mathbf{G}$ and $\mathbf{H}$ ) A very large macular soft druse from an 81-year-old female donor. Despite the difference in sizes and shapes of the drusen, the amyloid oligomer cores remain $10-15 \mu \mathrm{m}$ in size. Scale bars: $10 \mu \mathrm{m}$.

peptide, it has been shown to also detect toxic oligomers made from a variety of other amyloidogenic proteins (24) such as $\alpha$-synuclein, islet amyloid polypeptide (IAPP), prion 106-126 peptide, polyglutamine, lysozyme, human insulin, and, as recently demonstrated, the yeast prion protein (36). Remarkably, this antibody does not show reactivity (as demonstrated by dot blots and ELISA) with soluble monomeric protein, soluble low-molecular weight oligomers, or fibrils (24). It has been suggested, therefore, that the nonfibrillar amyloid oligomers from different proteins exhibit common structural features (24). Notably, the antibody also exhibits a strongly protective effect against oligomer-induced toxicity, indicating that oligomers do indeed represent a toxic species (24).

The utility of this generic anti-amyloid oligomer antibody has been established in immunocytochemical studies as well. For example, through this antibody, the presence of nonfibrillar oligomers has been demonstrated in AD-affected brains (24). These toxic oligomers were found to be in close proximity to senile plaques, yet they have shown a distinct localization from the fibrillar plaque region, perhaps indicating the initial stage of amyloid fibril deposition (24). Furthermore, the same antibody was used in immunocytochemical studies to identify amyloid oligomers in the above-mentioned study on desmin-related cardiomyopathy (35).

In the present study, by using the anti-oligomer antibody, we detected the toxic oligomers within drusen-containing donor eyes but not in control eyes without drusen. The presence of amyloid oligomers suggests that the underlying pathogenesis in AMD could be related to that of amyloid diseases.

\section{Results}

Immunofluorescent microscopy revealed the presence of amyloid oligomers in distinct areas of eyes that contained drusen. Antibody reactivity was most frequently observed centrally within drusen, wherein the fluorescent signal typically accumulated in close proximity to the inner collagenous layer of the Bm and formed a distinct subdomain. These structures, which we refer to as amyloid oligomer cores, did not vary appreciably in size, even as the size of the drusen varied. In the smallest drusen $(<20 \mu \mathrm{m})$, the amyloid oligomer cores comprised the majority of the drusen content (Figure 1, A and B). In larger drusen, the cores remained at approximately $15 \mu \mathrm{m}$ in diameter and retained the same spatial relationship, abutting the $\mathrm{Bm}$ whether they had the appearance of hard drusen (Figure 1, C-F) or macular soft drusen (Figure 1, $\mathrm{G}$ and $\mathrm{H}$ ). While many of the cores were spherical (e.g., Figure 1, B and D), others appeared more elongated in shape (e.g., Figure 1, F and $\mathrm{H})$. These data suggest that the oligomer cores may occur at 

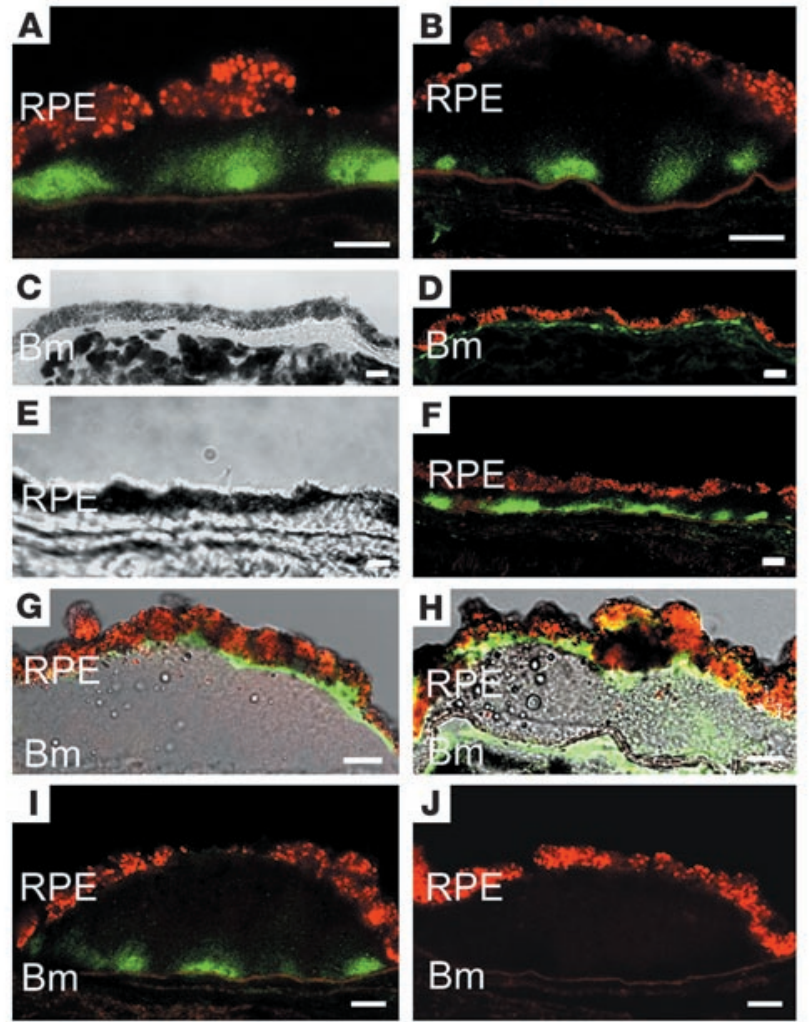

an early point during drusen biogenesis but do not appear to grow as drusen become larger.

Although the size of the amyloid oligomer cores appeared to be restricted, the number of cores per druse did vary. Larger drusen in particular were sometimes observed to contain several amyloid oligomer cores (Figure 2, A, B, and I), suggesting that these drusen may have formed from a coalescence of smaller drusen. While not all drusen were observed to contain amyloid oligomer cores, given the small size of the cores relative to the larger drusen, many were likely to be out of the plane of section and thus not detectable. Table 1 summarizes the results obtained from the eyes of 19 individuals. Strikingly, anti-oligomer antibody reactivity was observed in all eyes that contained drusen, but was not observed in eyes from age-matched controls or from those of young donors that did not contain drusen. These data establish a direct correlation between the presence of amyloid oligomers and drusen and suggest a possible role for oligomer cores in drusen biogenesis.

Besides the presence of amyloid oligomer cores within drusen, oligomer staining was also observed at the $\mathrm{Bm}$ in some cases, especially where it appeared to be thickened (Figure 2, C and D) and below the $\mathrm{Bm}$ in basal linear deposits (Figure 2, E-H). Occasional staining within the RPE was also observed (Figure $2 \mathrm{H}$ ). Staining was not observed in the neural retina (data not shown). Thus, antibody reactivity is also associated with additional pathological changes that are characteristic of AMD.

Specificity of the anti-oligomer antibody is exhibited in Figure 2, I and J, which depict serial sections obtained from the same druse. This druse contained several foci of anti-oligomer reactivity (Figure 2I). Staining was not seen when the section was incubated without the primary antibody (data not shown), nor when the antibody was preincubated with nonfibrillar oligomers made from the

\section{Figure 2}

Presence of amyloid oligomers in drusen and thickened Bm. Amyloid oligomer reactivity was visualized with fluorescein (green), and lipofuscin autofluorescence was visualized using the Cy3 channel (red). Multiple amyloid oligomer cores were sometimes observed in large drusen (A and $\mathbf{B}$ ), as if a large druse may have formed from the fusion of several smaller drusen. The amyloid oligomer cores retained their size and relative positions within the druse and in proximity to the $\mathrm{Bm}$. Within eyes that contained drusen, the oligomers occasionally accumulated above the $\mathrm{Bm}$, in the form of basal linear (C and $\mathbf{D}$ ) or basal laminar $(\mathbf{E}-\mathbf{H})$ deposits, particularly in instances where the $\mathrm{Bm}$ appeared to be thickened. $(\mathbf{H})$ Staining within RPE cells was also observed. $\mathbf{C}$ and $\mathbf{D}$ are differential interference contrast images of $\mathbf{D}$ and $\mathbf{F}$, respectively. (I and $\mathbf{J}$ ) Specificity of the antibody in cryosections is demonstrated in adjacent sections of a large druse. (I) Multiple amyloid oligomer cores were visualized through use of the anti-oligomer antibody. (J) Reactivity was eliminated when the primary antibody was preincubated with amyloid oligomers synthesized from the $A \beta_{1-40}$ peptide. Scale bars: $10 \mu \mathrm{m}$.

$\mathrm{A} \beta_{1-40}$ peptide (Figure $2 \mathrm{~J}$ ). It should be noted that the nonfibrillar oligomers made from $A \beta_{1-40}$ and $A \beta_{1-42}$ are structurally similar with regard to their immunoreactivity with the anti-oligomer antibody and their cellular toxicity (24). The difference lies in the kinetics of their formation: $A \beta_{1-42}$ forms oligomers on a faster time scale than does $A \beta_{1-40}(24,37)$. We performed ELISA in order to further test the specificity of the anti-oligomer antibody in tissue homogenates prepared from the neural retina or from the underlying tissue containing drusen/RPE/Bm. In vitro synthesized nonfibrillar $A \beta_{1-40}$ oligomers served as a positive control. As shown in Figure 3A, a dose-dependent reactivity to increasing amounts of $A \beta_{1-40}$ oligo-

Table 1

Oligomer reactivity is specific for drusen-containing tissue

\begin{tabular}{|c|c|c|c|c|}
\hline Age & Sex & Ocular history & Drusen & Oligomers \\
\hline \multicolumn{5}{|c|}{ Eyes with drusen } \\
\hline 87 & M & No data & + & + \\
\hline 86 & M & Unremarkable & + & + \\
\hline 88 & $\mathrm{~F}$ & AMD & + & + \\
\hline 96 & $\mathrm{~F}$ & No data & + & + \\
\hline 87 & $\mathrm{~F}$ & No data & + & + \\
\hline 94 & $\mathrm{~F}$ & No data & + & + \\
\hline 92 & $\mathrm{M}$ & Unremarkable & + & + \\
\hline 92 & $\mathrm{~F}$ & Pseudophakia & + & + \\
\hline 75 & $\mathrm{~F}$ & AMD & + & + \\
\hline 83 & M & Intraocular lens surgery & + & + \\
\hline 82 & $\mathrm{~F}$ & AMD & + & + \\
\hline 98 & M & AMD & + & + \\
\hline 88 & $\mathrm{~F}$ & Cataract & + & + \\
\hline 77 & M & Unremarkable & + & + \\
\hline \multicolumn{5}{|c|}{ Eyes without drusen } \\
\hline 87 & M & Unremarkable & - & - \\
\hline 81 & M & Cataract & - & - \\
\hline 56 & $\mathrm{~F}$ & Unremarkable & - & - \\
\hline 21 & $\mathrm{~F}$ & Unremarkable & - & - \\
\hline Fetus & & No data & - & - \\
\hline
\end{tabular}

Whole eyes from 19 donors were screened by confocal microscopy for the presence of amyloid oligomers. Oligomer reactivity was observed only when drusen were present. No reactivity was observed in agematched control eyes without drusen or in eyes from young donors that did not contain drusen. 

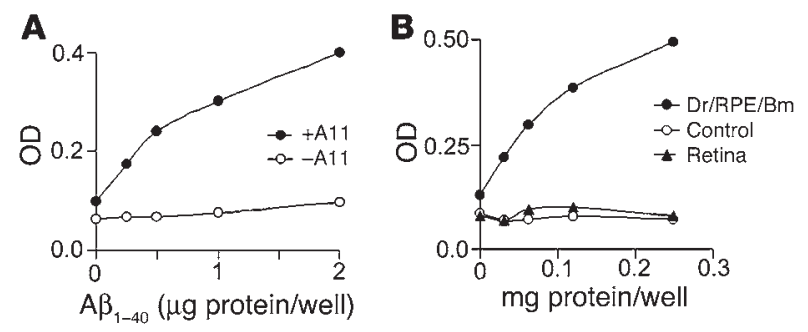

Figure 3

ELISA of retinal extracts using the A11 anti-oligomer antibody. (A) Increasing amounts of oligomers made from the $A \beta_{1-40}$ peptide resulted in a dose-dependent response when incubated with the A11 anti-oligomer-specific antibody (filled circles). Little or no reactivity was observed when the $A \beta_{1-40}$ oligomers were incubated without the primary antibody (open circles). (B) Dose-dependent reactivity was observed when the A11 antibody was incubated with increasing amounts of extract prepared from dissected drusen/RPE/Bm tissue from a 76-year-old male donor (filled circles). Little or no reactivity was observed when the primary antibody was omitted (open circles). Extracts prepared from the neural retina (filled triangles) of the same donor eye did not show a dose-dependent response when incubated with the A11 antibody. Dr, drusen.

mers was observed in the presence of the oligomer-specific antibody, but not when the antibody was omitted. Comparisons of antibody reactivity were also made between extracts prepared from the neural retina and from the tissue containing drusen/RPE/Bm (Figure 3B). A dose-dependent reactivity was observed with extracts prepared from drusen-containing tissue, whereas no reactivity was observed in the absence of the primary antibody. Little reactivity was observed with extracts prepared from the neural retina of the same donor eye. Thus the positive signal seen in drusen appears to be highly specific for amyloid oligomers.

The data presented thus far support the notion that amyloid oligomers are present in drusen. Further inspections of the sections at higher magnifications using laser scanning confocal microscopy revealed a punctate pattern of small vesicular structures that increased in density toward the center of the amyloid oligomer core (Figure 4A). Occasionally, a decreasing gradation of the punctate pattern appeared to extend toward the RPE (Figure $4 \mathrm{~B})$. In other instances, the vesicular structures were observed

\section{Figure 4}

Morphology of amyloid oligomer cores in drusen at higher magnification. (A-C) Confocal micrographs of drusen. Amyloid oligomer cores are labeled with fluorescein (green), and lipofuscin autofluorescence in the RPE is visualized in red (Cy3 channel). (A) Amyloid oligomer cores seemed to consist of an aggregate of small vesicular structures (arrowheads) that increased in density toward the center. (B) Some of these vesicular structures appeared to extend toward the RPE with diminishing density (arrowheads). (C) Occasionally, the amyloid oligomer cores penetrated through the $\mathrm{Bm}$ and extended toward the choroid (arrowhead). (D) Ultrastructure of an amyloid oligomer core is depicted in an immunogold-labeled electron micrograph (inset), wherein gold particles decorate vesicular structures that are heterogeneous in size. The highest density of gold particles seen in $\mathbf{D}$ was from the region above the $\mathrm{Bm}$ (rectangle). Scale bars: $100 \mathrm{~nm}$ (D, inset). Magnification, $\times 3,000(\mathbf{A}), \times 2,000(\mathbf{B}-\mathbf{D})$. to penetrate through the layers of the Bm (Figure 4C). Together, these data suggest that the amyloid oligomers could be trafficked between the RPE cells, the drusen, and the choroid.

In order to confirm the vesicular nature of the cores, we first used indirect immunofluorescence to identify a druse that contained an amyloid oligomer core. An adjacent serial section was then prepared for immunogold labeling, followed by electron microscopy (Figure 4D). As judged by distribution of the gold particles, these studies did indeed verify that the amyloid oligomers were associated with vesicular structures. Again, these vesicular structures appeared to be more concentrated near the Bm, although similar structures were occasionally labeled within the apical aspect of the druse closer to the RPE as well (data not shown).

The structures of the amyloid oligomer cores appear to be similar in some respects to dendritic cell processes, as reported by Hageman et al. (8). Double staining with the anti-oligomer antibody (green, FITC) was performed in conjunction with anti-HLA-DR (red, Texas red) in order to determine whether the immunoreactivities colocalized (Figure 5, A-D). Strong HLA-DR reactivity was seen within the drusen (Figure $5 \mathrm{~B}$ ) or beneath the $\mathrm{Bm}$, where it appeared to penetrate into the druse and come into close proximity to the oligomer core (Figure 5C) and even completely surround it (Figure 5D). In some instances, HLA-DR reactivity appeared to be in close proximity to the amyloid oligomer core (Figure 5B). Upon closer inspection, it was clear that the immunofluorescent signals did not overlap (Figure 5, C and D). Thus the amyloid oligomer cores are distinct structures from the HLA-DR-positive dendritic cell processes described above.

Double staining was also performed on drusen sections to visualize oligomer cores and vitronectin, an acute phase protein that is a major component of drusen (38) (Figure 5, E-H). All drusen stained positively for vitronectin, whereas oligomer cores were present only in a subset of drusen (e.g., Figure 5F). Vitronectin tends to have heterogeneous labeling patterns. In drusen that reacted positively for both oligomer cores and vitronectin, no overlap in their signals was observed. To ascertain whether the oligomer cores are assembled from $A \beta$, sections containing drusen were costained for these 2 components (Figure 5, I-L). Most drusen contained either the $A \beta$ assemblies or oligomer cores, but not both. Consistent with previous reports, $A \beta$ reactivity was associated with vesicular structures within drusen (Figure 5, $\mathrm{K}$ and $\mathrm{L}$ ). In 1 druse that reacted with both antibodies, the fluorescent signals did not colocalize: the
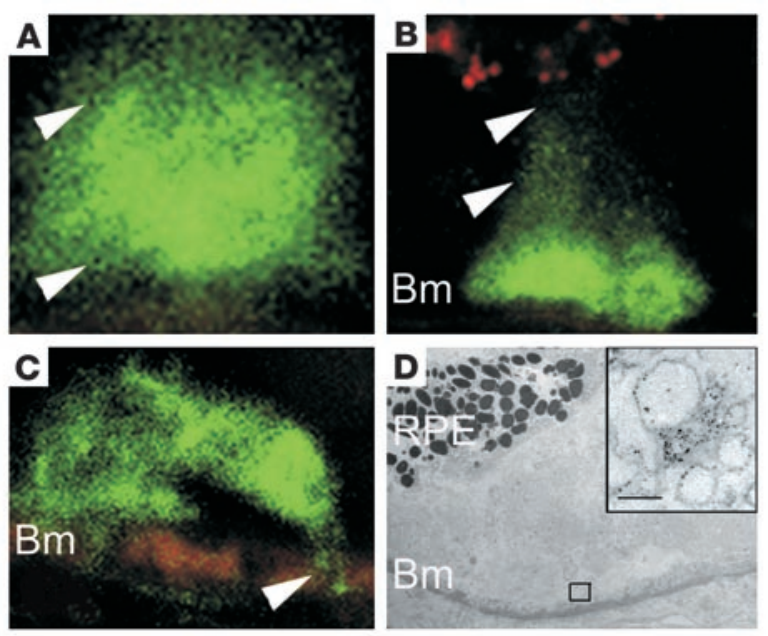

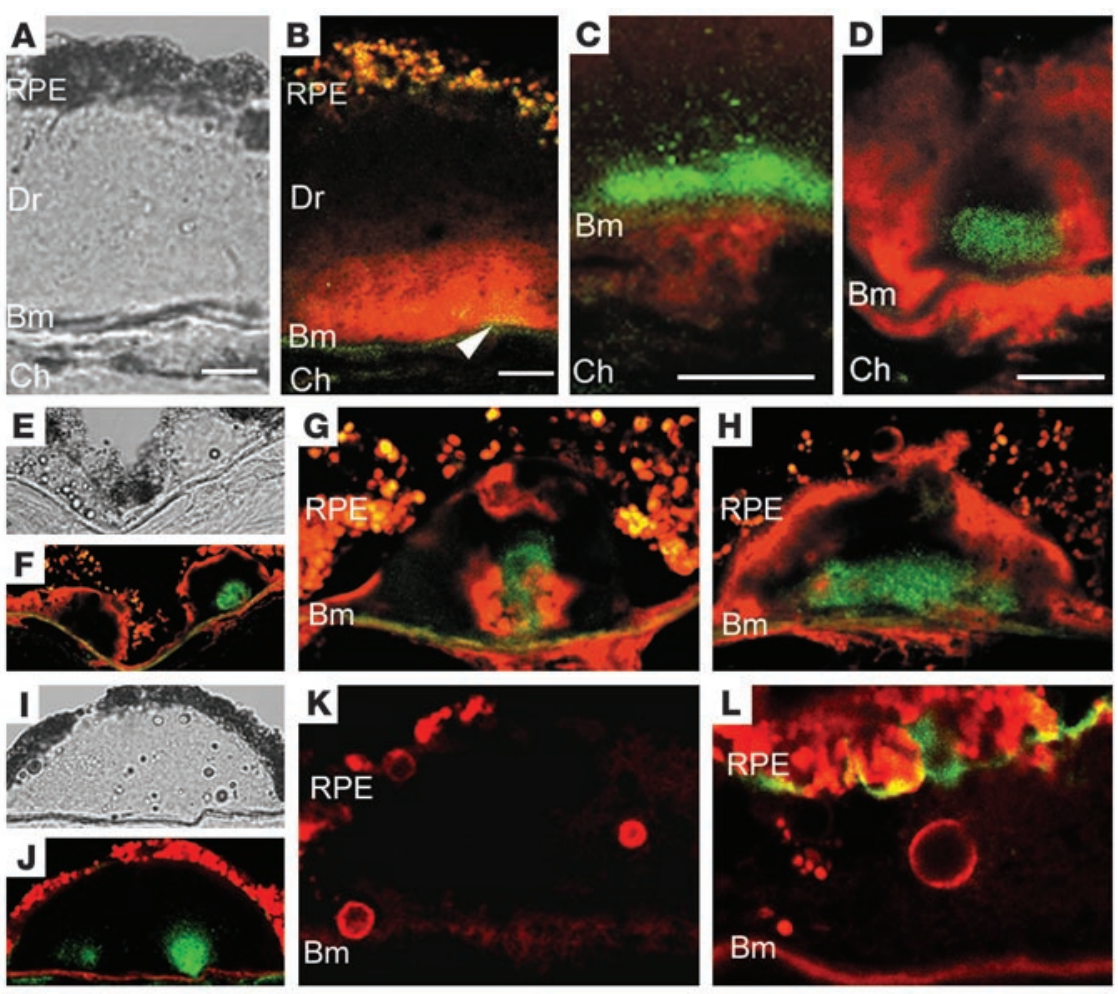

Figure 5

Codistribution of amyloid oligomer cores and other known drusen components. (A, E, and I) Differential interference contrast images. (B-D, F-H, and $\mathbf{J}-\mathbf{L}$ ) Confocal fluorescence images; amyloid oligomer cores were labeled with fluorescein (green). (A and B) Both antigens were present in a large druse, wherein the amyloid oligomer core was enveloped within the HLA-DR reactive region (labeled with Texas red). (C and $\mathbf{D})$ At higher magnification, it is clear that the amyloid oligomer core and HLA-DR reactive subdomain did not colocalize in these drusen. (C) In one instance, the HLA-DR reactive region, perhaps reflecting a dendritic cell process, was observed as originating from the choroid (Ch), coming in close proximity to the $\mathrm{Bm}$, and contacting the condensation of vesicular structures that represent the amyloid oligomer core. (D) In another instance, HLA-DR reactivity was observed as encompassing the choroid, the Bm, and the druse. Within the druse, HLA-DR reactivity appeared to surround the oligomer core, with no indication of colocalization. Similarly, no colocalization was observed with vitronectin $(\mathbf{F}-\mathbf{H})$ or $A \beta(\mathbf{J}-\mathbf{L})$, both labeled with Texas red (red). Lipofuscin autofluorescence within RPE is also visualized in the Cy3 channel (red). Scale bars: $10 \mu \mathrm{m}$. Magnification, $\times 250(\mathbf{E}, \mathbf{F}, \mathbf{I}$, and $\mathbf{J}), \times 1,500(\mathbf{G}), \times 1,000(\mathbf{H})$, and $\times 2,000(\mathbf{K}$ and $\mathbf{L})$.

to RPE cells may have a negative impact on the physiology of these cells during drusen biogenesis and in AMD.

\section{Discussion}

Nonfibrillar oligomeric structures made from amyloidogenic proteins or peptides are thought to contribute to the pathogenesis of amyloid diseases. Such structures can be detected in tissue sections in situ by a recently developed conformation-specific, but not sequence-specific, antibody (24). Through the use of this antibody, we demonstrated the presence of amyloid oligomers in drusencontaining eyes and eyes that have been clinically diagnosed with atrophic AMD. Importantly, no reactivity was observed in control eyes without drusen, which suggests that the formation of amyloid oligomers is a diseasespecific process. Since nonfibrillar amyloid oligomers demonstrated toxicity toward cultured primary human RPE cells, they may contribute to their demise during the disease process. Thus, AMD and amyloid diseases appear to share similar protein misfolding events and may share common pathogenic pathways as well.

One commonality is the discovery that spherical $A \beta$ assemblies as well as other proinflammatory proteins commonly seen in $\mathrm{AD}$ plaques are also present in drusen $(9,10)$. In particular, Anderson et al. showed that a single druse may contain no $A \beta$ structures or a large number of them, ranging in diameter from 0.25 to $10 \mu \mathrm{m}$ and displaying highly organized concentric layers when viewed under an electron microscope (5). Thus, the $\mathrm{A} \beta$ assemblies reported by Anderson et al. are structurally distinct from the oligomerassociated vesicles due to differences in their size, shape, and distribution. Indeed, our data show that they did not colocalize in drusen. It is important to note, however, that the epit-

amyloid oligomer reactivity was associated with the RPE, whereas the $\mathrm{A} \beta$ reactivity decorated spherical structures within the druse (Figure 5L). Thus amyloid oligomers do not appear to colocalize with many of the known drusen components.

Different tissue or cultured cell types show varying susceptibility to the toxicity of amyloid aggregates (39). We sought to examine whether amyloid oligomers are toxic to RPE cells, given their close proximity to each other in eyes that contain drusen. As expected, oligomeric, but not soluble monomeric, IAPP and $\alpha$-synuclein exerted toxicity toward human primary RPE cells and SH-SY5Y human neuroblastoma cells (Figure 6A). Similarly, oligomers made from $A \beta_{1-40}$ were toxic to human primary RPE cells in culture. This toxicity was largely blocked when the A11 anti-oligomer antibody was included in the incubation mixture (Figure 6B). These results confirm that nonfibrillar oligomers made from amyloidogenic protein and peptides are toxic to human primary RPE cells. Thus, the presence of amyloid oligomers in close proximity ope for $\mathrm{A} \beta$ may have been masked within the oligomeric structure, as is the case when $A \beta$ monomers are transformed into amyloid fibrils (40). Therefore, we cannot preclude the possibility that the oligomeric cores in drusen are made up of $A \beta$. Another drusen subdomain, described by Mullins and Hageman (41), is composed of core-like structures that exhibit Arachea bypogea agglutinin reactivity. Although these structures to some extent resemble the oligomeric structures described herein, they range in diameter from 5 to $38 \mu \mathrm{m}$, whereas the amyloid oligomer cores are typically $10-15 \mu \mathrm{m}$. It appears then that the oligomeric structures discussed here differ distinctively from the substructures within drusen that had been described previously. Composition of the oligomeric structures within drusen has yet to be determined and is under investigation.

Our data have yielded further evidence that AMD and amyloid diseases share common pathogenic pathways, although amyloid fibrils have not been observed in drusen. Amyloid protein-related toxicity in the absence of fibrils had been observed in the past. For 

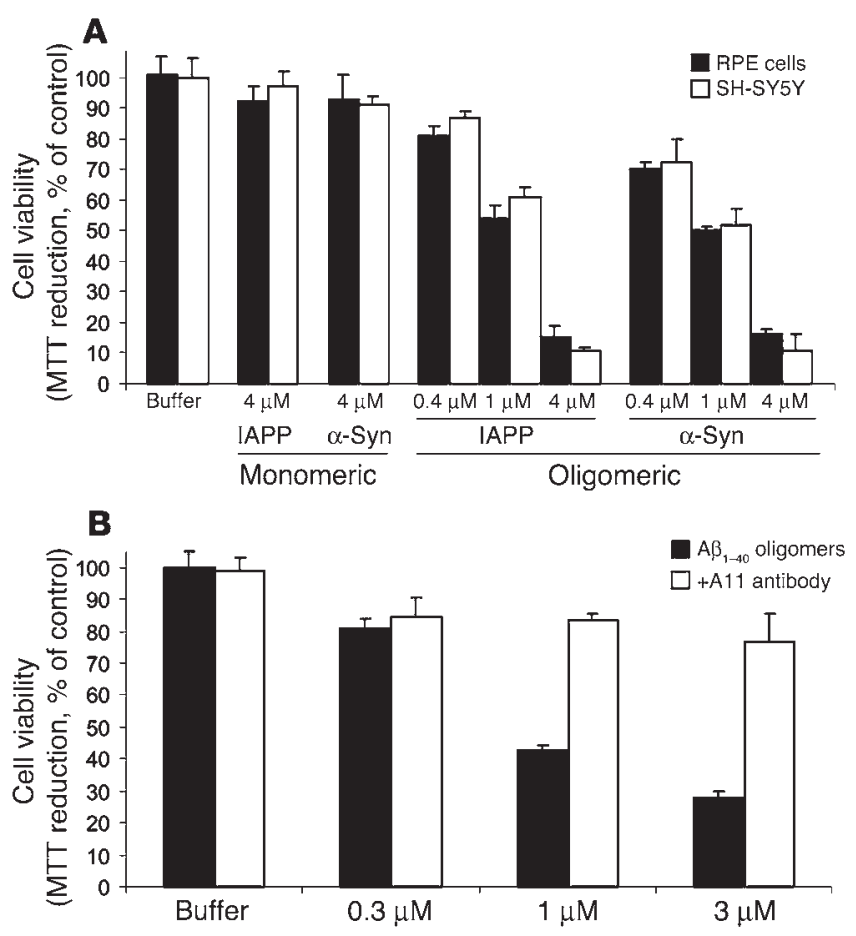

Figure 6

Toxicity of nonfibrillar amyloid oligomers to human primary RPE cells. (A) Amyloid oligomers were toxic to cultured SH-SY5Y human neuroblastoma cells (white bars) and human primary RPE cells (black bars). Oligomeric forms of IAPP and $\alpha$-synuclein ( $\alpha$-Syn), but not soluble monomers, demonstrated toxicity. Cell viability was assessed by MTT reduction. (B) Increasing amounts of amyloid oligomers made from $A \beta_{1-40}$ also showed a dose-dependent toxicity to cultured human primary RPE cells (black bars). This toxicity was largely blocked by adding equal molars of the A11 anti-oligomer antibody (white bars). Error bars represent SD; $n=3$.

example, in a transgenic mouse model for $\mathrm{AD}$, overexpression of the human wild-type $\beta$-amyloid precursor protein leads to learning deficits and $A \beta$ deposition without amyloid plaque formation (42). In the case of a mouse model for PD, it has been shown that overexpression of wild-type $\alpha$-synuclein results in motor abnormalities and the formation of $\alpha$-synuclein-containing, nonfibrillar inclusions (43). In transgenic rats expressing human IAPP that served as an animal model for type 2 diabetes, apoptosis of pancreatic islet cells did not correlate with amyloid formation (33). These results indicate that the presence of amyloid fibrils is not a prerequisite for pathogenesis and implicate the toxic nonfibrillar oligomers as an underlying cause of cell loss (30). Thus, AMD and desmin-related cardiomyopathy (35) might be just 2 of several diseases that are related to amyloid diseases, yet do not exhibit noticeable amyloid fibril deposition.

Although it is not obvious why amyloid fibrils are difficult to detect in drusen, the rate at which oligomers and fibrils are turned over are likely to be of importance. As mentioned above, amyloid fibril formation is a stepwise process, and the overall yield of oligomers and fibrils depends upon the underlying kinetics of each step. Thus, 2 possible explanations for the low degree of fibril deposition are slow rates of fibril formation or fast rates of clearance. It is known that rates of amyloid fibril formation are largely dictated by experimental conditions, and biochemical data suggest that under appropriate conditions the stability of oligomers can be maintained for extended periods of time $(44,45)$. The stability of the oligomers in the drusen may also be stabilized through interacting with other proteins. In addition, it is conceivable that oligomers might be cleared out of drusen before they can be converted into fibrils. Although the present study provides no direct evidence of such clearance, the ability of oligomeric structures to penetrate through the Bm suggests this possibility.

Recent immunocytochemical data on HLA-DR reactivity in drusen suggest the presence of dendritic cell processes in drusen (8). Dendritic cells are antigen-presenting cells that take up foreign substances and, in principle, may facilitate the clearance of amyloid oligomers. Indeed, our studies show the presence of HLADR-reactive structures in drusen similar to that reported by Hageman et al. (8). These putative dendritic cell processes appear more frequently in and around the drusen than the amyloid oligomers, and they were sometimes found in close proximity to the amyloid oligomer cores. They do not, however, appear to colocalize. Future studies should enable us to determine whether a relationship exists between the dendritic cells and the amyloid oligomer cores.

Our data add to the growing list of evidence that reveals similarities between AMD and amyloid diseases (4-7). It is particularly noteworthy that proinflammatory proteins have been identified in the extracellular deposits associated with these diseases. Evidence of complement activation has been observed within certain RPE cells, small drusen, and large, soft drusen that are present in aging eyes as well as in AMD eyes (10). This observation has led to the hypothesis that aberrant immune reactions may play a role in drusen biogenesis. Notably, a polymorphism in complement factor $\mathrm{H}$, a key regulator of complement activation, has recently been identified as a major risk factor for AMD (12-15). This finding has placed a significant focus on the role of complement activation in the pathogenesis of AMD: what are the factors that lead to the activation of the immune response? Here we report the presence of amyloid oligomers in a similar distribution of drusen, RPE cells, and basal deposits. It is noteworthy that these oligomers have been implicated in the pathogenesis of amyloid diseases due to their demonstrated toxicity toward cells. It is possible that the presence of oligomers in close proximity to RPE cells may compromise their function, leading to activation of the complement cascade and formation of drusen.

In summary, the presence of amyloid oligomers in drusen suggests that AMD and amyloid diseases share commonalities with respect to protein misfolding and pathogenesis. AMD and desminrelated cardiomyopathy (35) may well come to represent the first examples of a new class of amyloid disease in which oligomeric intermediates, rather than mature amyloid fibrils, accumulate.

\section{Methods}

Human tissue. Intact human donor eyes were obtained from the Oregon Lions Sight and Hearing Foundation and the Alzheimer Disease Research Center (ADRC) Neuropathology Core of the University of Southern California. Eyes from 19 individuals were examined, 4 of which had documented clinical histories of AMD (Table 1). All eyes were kept at $4{ }^{\circ} \mathrm{C}$ and processed at less than 24 hours postmortem. Fixation was avoided since it would have interfered with antigen detection using the anti-oligomerspecific antibody. After removing the anterior pole, the retina was peeled off and the posterior pole of the eyeball was examined under a dissecting microscope (MZ125; Leica Microsystems) for the presence of drusen. All areas containing drusen were included. Tissue was cut into $1-\mathrm{cm} \times 0.5-\mathrm{cm}$ 
rectangles, using a coated stainless steel razor blade, and embedded in OCT (Tissue-Tek; Sakura Finetech).

Confocal immunofluorescence microscopy. Frozen embedded tissue was sectioned on a cryostat (CM 3050S; Leica Microsystems) at $-20^{\circ} \mathrm{C}$. Frozen sections $8-10 \mu \mathrm{m}$ thick were collected on precleaned Superfrost plusslides (VWR International), air dried for 30 minutes, and stored at $-20^{\circ} \mathrm{C}$. Immunocytochemical studies using the anti-oligomer-specific antibody were performed as described previously (24). Briefly, sections were blocked overnight at $4^{\circ} \mathrm{C}$ in blocking solution (phosphate-buffered saline containing $2 \% \mathrm{BSA}$ and $2 \%$ goat serum) and incubated the following day with affinity-purified anti-oligomer-specific antibody $(1.6 \mathrm{mg} / \mathrm{ml})$ for 1 hour at room temperature. Sections were then washed and incubated with a fluorescein-conjugated goat anti-rabbit antibody (1:100; Vector Laboratories) for 1 hour at room temperature. In order to detect oligomers and HLA-DR or drusen components such as vitronectin and $A \beta$, sections were processed as described above and incubated with mouse anti-human HLA-DR antibody $(0.5 \mathrm{mg} / \mathrm{ml}, \mathrm{BD}$ Biosciences - Pharmingen $)$, mouse anti-vitronectin antibody (1:200, BioSource International), or mouse anti- $\mathrm{A} \beta$ antibody (1:100, 4G8; Signet Laboratories Inc.), which is directed against residues 17-24 of the $A \beta$ peptide. Digital images of immunostained sections were acquired on an LSM 510 Zeiss laser scanning confocal microscope.

Electron microscopy. Prefibrillar oligomers were first identified in frozen sections using immunofluorescence. Adjacent serial sections known to contain oligomers were incubated with the anti-oligomer antibody, and subsequently with $5 \mathrm{~nm}$ gold-conjugated goat anti-rabbit antibody (Ted Pella Inc.). The sections were washed and pre-embedded in $4 \%$ agarose. Agarose-embedded sections were then briefly fixed in $\mathrm{OsO}_{4}$, dehydrated in increasing concentrations of ethanol, infiltrated with epoxy resin, and sectioned at $70 \mathrm{~nm}$ using an ultramicrotome (Ultracut UCT; Leica Microsystems) for electron microscopy. Images were obtained using a transmission electron microscope (EM10; Zeiss).

Preparation of soluble $A \beta$ oligomers. $A \beta$ oligomers were prepared as described previously (26). Briefly, $1.0 \mathrm{mg} \mathrm{A} \beta$ was dissolved in $400 \mu \mathrm{l}$ 1,1,1,3,3,3-hexafluoro-2-propanol (HFIP) for 10 minutes at room temperature. Aliquots (100 $\mu \mathrm{l}$ ) of the solution were added to $900 \mu \mathrm{l}$ double-distilled $\mathrm{H}_{2} \mathrm{O}$ in siliconized Eppendorf tubes. After 10 minutes of incubation the samples were centrifuged for 15 minutes at $14,000 \mathrm{~g}$, and the supernatant fraction was transferred to a new siliconized tube. The HFIP was evaporated by blowing under an $\mathrm{N}_{2}$ stream for 5-10 minutes. The samples were then stirred at $500 \mathrm{rpm}$ using a Teflon coated micro stir bar for $24-48$ hours at room temperature. Aliquots were taken at 6 - to 12 -hour intervals to check for the presence of spherical oligomers.

ELISA. ELISA was performed with tissue homogenates and oligomers made from the $A \beta$ peptide. To process eye tissue samples, neural retina was peeled off the underlying RPE/Bm/choroid complex at the posterior eye pole. Isolated tissues (neural retina or the underlying RPE/Bm/ choroid complex) were homogenized using a tip sonicator (Microson) in double-distilled $\mathrm{H}_{2} \mathrm{O}$ and centrifuged, and supernatant was collected. ELISA was performed using the anti-oligomer-specific antibody as described by Kayed and colleagues (24). Briefly, samples were diluted in coating buffer $(0.1 \mathrm{M}$ sodium bicarbonate) and added to wells of a 96-well microplate (BD). After 2 hours of incubation at $37^{\circ} \mathrm{C}$, samples were blocked for 2 hours at $37^{\circ} \mathrm{C}$ with $3 \%$ BSA tris-buffered saline with $0.1 \%$ Triton X-100. Anti-oligomer antibody (100 $\mu 1,1: 2500)$ was added and incubated at $37^{\circ} \mathrm{C}$ for 1 hour, prior to incubation with $100 \mu \mathrm{l}$ of horseradish peroxidase-conjugated anti-rabbit IgG for 1 hour at $37^{\circ} \mathrm{C}$. Subsequent to development with $3,3^{\prime}, 5,5^{\prime}$-tetramethylbenzidine, the reaction was stopped with $100 \mu \mathrm{l} 1 \mathrm{M} \mathrm{HCl}$, and plates were read at $450 \mathrm{~nm}$ (Benchmark Plus; Bio-Rad Laboratories).

Cell viability assay. Cell viability was assessed spectrophotometrically using a 3-[4,5-dimethylthiazol-2-yl]-2,5-diphenyl tetrazolium bromidebased (MTT-based) assay (Sigma-Aldrich). RPE cells isolated from human fetal eyes were obtained from Advanced Bioscience Resources Inc. The cells were maintained in DMEM supplemented with $2 \mathrm{mM}$ L-glutamine, 100 $\mathrm{U} / \mathrm{ml}$ penicillin, $100 \mu \mathrm{g} / \mathrm{ml}$ streptomycin, and $10 \%$ fetal bovine serum at $37^{\circ} \mathrm{C}$. Third to fourth passage cells were seeded at 10,000 cells per well in a 96-well plate and grown for 3-4 days to approximately $90 \%$ confluence. SH-SY5Y human neuroblastoma cells were maintained in DMEM with $10 \mathrm{nM}$ HEPES, $10 \%$ fetal bovine serum, $4 \mathrm{mM}$ glutamine, penicillin $(200 \mathrm{U} / \mathrm{ml})$, and streptomycin $(200 \mu \mathrm{g} / \mathrm{ml})$ in $5 \% \mathrm{CO}_{2}$ at $37^{\circ} \mathrm{C}$. They were differentiated and plated as previously described (24). Prior to the toxicity assay, media was replaced with indicated concentrations of $A \beta$ oligomers alone or with equal molars of the A11 anti-oligomer antibody dissolved in phenol red-free DMEM. The conditions were carried out in triplicate. After 4 hours, MTT dissolved in DMEM was added to the cells and incubated for an additional 4 hours. Insoluble crystals were dissolved by adding MTT solubilization solution (10\% Triton X-100, $0.1 \mathrm{~N} \mathrm{HCl}$ in anhydrous isopropanol), and absorbance was measured at $570 \mathrm{~nm}$.

\section{Acknowledgments}

We thank Ruth Phinney and Rory Dunaway from the Oregon Lions Sight and Hearing Foundation for their help in providing to us whole eyes and eyes with a history of AMD according to our specifications. We also thank the ADRC Neuropathology Core, University of Southern California, for providing us with whole eyes for this study. This work was supported by grants from the Arnold and Mabel Beckman Foundation (to R. Langen and J. Chen), the Larry L. Hillblom Foundation (to C.G. Glabe, R. Langen, and J. Chen), and the NIH (grant NIA AG05142, to ADRC) and by a Vision Core Grant to Doheny Eye Institute (EY03040).

Received for publication June 3, 2005, and accepted in revised form November 22, 2005.

Address correspondence to: Jeannie Chen, Zilkha Neurogenetic Institute, 1501 San Pablo Street, ZNI-227, Los Angeles, California 90033, USA. Phone: (323) 442-4479; Fax: (323) 442-4433; E-mail: jeannie@usc.edu.
1. Klein, R., Peto, T., Bird, A., and Vannewkirk, M.R. 2004. The epidemiology of age-related macular degeneration. Am. J. Ophthalmol. 137:486-495.

2. Johnson, P.T., et al. 2003. Drusen-associated degeneration in the retina. Invest. Ophthalmol. Vis. Sci. 44:4481-4488.

3. Abdelsalam, A., Del Priore, L., and Zarbin, M.A. 1999. Drusen in age-related macular degeneration: pathogenesis, natural course, and laser photocoagulationinduced regression. Surv. Ophthalmol. 44:1-29.

4. Crabb, J.W., et al. 2002. Drusen proteome analysis: an approach to the etiology of age-related macular degeneration. Proc. Natl. Acad. Sci. U. S. A.
99:14682-14687.

5. Anderson, D.H., et al. 2004. Characterization of beta amyloid assemblies in drusen: the deposits associated with aging and age-related macular degeneration. Exp. Eye Res. 78:243-256.

6. Mullins, R.F., Russell, S.R., Anderson, D.H., and Hageman, G.S. 2000. Drusen associated with aging and age-related macular degeneration contain proteins common to extracellular deposits associated with atherosclerosis, elastosis, amyloidosis, and dense deposit disease. FASEB J. 14:835-846.

7. Malek, G., et al. 2005. Apolipoprotein E alleledependent pathogenesis: a model for age-related retinal degeneration. Proc. Natl. Acad. Sci. U. S. A. 102:11900-11905.

8. Hageman, G.S., et al. 2001. An integrated hypothesis that considers drusen as biomarkers of immunemediated processes at the RPE-Bruch's membrane interface in aging and age-related macular degeneration. Prog. Retin. Eye Res. 20:705-732.

9. Johnson, L.V., et al. 2002. The Alzheimer's Abetapeptide is deposited at sites of complement activation in pathologic deposits associated with aging and age-related macular degeneration. Proc. Natl. Acad. Sci. U. S. A. 99:11830-11835.

10. Johnson, L.V., Leitner, W.P., Staples, M.K., and 
Anderson, D.H. 2001. Complement activation and inflammatory processes in Drusen formation and age related macular degeneration. Exp. Eye Res. 73:887-896.

11. Ambati, J., et al. 2003. An animal model of agerelated macular degeneration in senescent Ccl-2- or Ccr-2-deficient mice. Nat. Med. 9:1390-1397.

12. Hageman, G.S., et al. 2005. From the cover: a common haplotype in the complement regulatory gene factor $\mathrm{H}$ (HF1/CFH) predisposes individuals to age-related macular degeneration. Proc. Natl. Acad. Sci.U.S. A. 102:7227-7232.

13. Edwards, A.O., et al. 2005. Complement factor $\mathrm{H}$ polymorphism and age-related macular degeneration. Science. 308:421-424.

14. Haines, J.L., et al. 2005. Complement factor $\mathrm{H}$ variant increases the risk of age-related macular degeneration. Science. 308:419-421.

15. Klein, R.J., et al. 2005. Complement factor $\mathrm{H}$ polymorphism in age-related macular degeneration. Science. 308:385-389.

16. Soto, C. 2003. Unfolding the role of protein misfolding in neurodegenerative diseases. Nat. Rev. Neurosci. 4:49-60.

17. Sunde, M., and Blake, C. 1997. The structure of amyloid fibrils by electron microscopy and X-ray diffraction. Adv. Protein Chem. 50:123-159.

18. Puchtler, H., Sweat, F., and Levine, M. 1962. On the binding of Congo red by amyloid. J. Histochem. Cytochem. 10:355-364.

19. LeVine, H., 3rd. 1999. Quantification of beta-sheet amyloid fibril structures with thioflavin T. Methods Enzymol. 309:274-284.

20. Krebs, M.R.H., Bromley, E.H.C., and Donald, A.M. 2005. The binding of thioflavin-T to amyloid fibrils: localisation and implications. J. Struct. Biol. 149:30-37.

21. Loffler, K.U., Edward, D.P., and Tso, M.O. 1995. Immunoreactivity against tau, amyloid precursor protein, and beta-amyloid in the human retina. Invest. Ophthalmol. Vis. Sci. 36:24-31.

22. Reixach, N., Deechongkit, S., Jiang, X., Kelly, J.W., and Buxbaum, J.N. 2004. Tissue damage in the amyloidoses: transthyretin monomers and nonnative oligomers are the major cytotoxic spe- cies in tissue culture. Proc. Natl. Acad. Sci. U. S. A. 101:2817-2822.

23. Kayed, R., et al. 2004. Permeabilization of lipid bilayers is a common conformation-dependent activity of soluble amyloid oligomers in protein misfolding diseases. J. Biol. Chem. 279:46363-46366.

24. Kayed, R., et al. 2003. Common structure of soluble amyloid oligomers implies common mechanism of pathogenesis. Science. 300:486-489.

25. Walsh, D.M., et al. 2002. Naturally secreted oligomers of amyloid beta protein potently inhibit hippocampal long-term potentiation in vivo. Nature. 416:535-539.

26. Klein, W.L., Krafft, G.A., and Finch, C.E. 2001. Targeting small A[beta] oligomers: the solution to an Alzheimer's disease conundrum? Trends Neurosci. 24:219-224.

27. Kirkitadze, M.D., Bitan, G., and Teplow, D.B. 2002. Paradigm shifts in Alzheimer's disease and other neurodegenerative disorders: the emerging role of oligomeric assemblies. J. Neurosci. Res. 69:567-577.

28. Lambert, M.P., et al. 1998. Diffusible, nonfibrillar ligands derived from Abeta1-42 are potent central nervous system neurotoxins. Proc. Natl. Acad. Sci. U. S. A. 95:6448-6453.

29. Anguiano, M., Nowak, R.J., and Lansbury, P.T., Jr. 2002. Protofibrillar islet amyloid polypeptide permeabilizes synthetic vesicles by a pore-like mechanism that may be relevant to type II diabetes. Biochemistry. 41:11338-11343.

30. Caughey, B., and Lansbury, P.T. 2003. Protofibrils, pores, fibrils, and neurodegeneration: separating the responsible protein aggregates from the innocent bystanders. Annu. Rev. Neurosci. 26:267-298.

31. Mucke, L., et al. 2000. High-level neuronal expression of abeta 1-42 in wild-type human amyloid protein precursor transgenic mice:synaptotoxicity without plaque formation. J. Neurosci. 20:4050-4058.

32. Hsia, A.Y., et al. 1999. Plaque-independent disruption of neural circuits in Alzheimer's disease mouse models. Proc. Natl. Acad. Sci. U. S. A. 96:3228-3233.

33. Butler, A.E., et al. 2004. Diabetes due to a progressive defect in beta-cell mass in rats transgenic for human islet amyloid polypeptide (HIP rat): a new model for type 2 diabetes. Diabetes. 53:1509-1516.
34. Moechars, D., et al. 1999. Early phenotypic changes in transgenic mice that overexpress different mutants of amyloid precursor protein in brain. J. Biol. Chem. 274:6483-6492.

35. Sanbe, A., et al. 2004. Desmin-related cardiomyopathy in transgenic mice: a cardiac amyloidosis. Proc. Natl. Acad. Sci. U. S. A. 101:10132-10136.

36. Shorter, J., and Lindquist, S. 2004. Hsp104 catalyzes formation and elimination of self-replicating Sup35 prion conformers. Science. 304:1793-1797.

37. Stine, W.B., Jr., Dahlgren, K.N., Krafft, G.A., and $\mathrm{LaDu}$, M.J. 2003. In vitro characterization of conditions for amyloid-beta peptide oligomerization and fibrillogenesis. J. Biol. Chem. 278:11612-11622.

38. Hageman, G.S., Mullins, R.F., Russell, S.R., Johnson, L.V., and Anderson, D.H. 1999. Vitronectin is a constituent of ocular drusen and the vitronectin gene is expressed in human retinal pigmented epithelial cells. FASEB J. 13:477-484.

39. Cecchi, C., et al. 2005. Insights into the molecular basis of the differing susceptibility of varying cell types to the toxicity of amyloid aggregates. J. Cell Sci. 118:3459-3470.

40. Miller, D.L., et al. 2003. Humoral immune response to fibrillar beta-amyloid peptide. Biochemistry. 42:11682-11692.

41. Mullins, R.F., and Hageman, G.S. 1999. Human ocular drusen possess novel core domains with a distinct carbohydrate composition. J. Histochem. Cytochem. 47:1533-1539.

42. Koistinaho, M., et al. 2001. Specific spatial learning deficits become severe with age in beta-amyloid precursor protein transgenic mice that harbor diffuse beta -amyloid deposits but do not form plaques. Proc. Natl. Acad. Sci. U. S. A. 98:14675-14680.

43. Masliah, E., et al. 2000. Dopaminergic loss and inclusion body formation in alpha-synuclein mice: implications for neurodegenerative disorders. Science. 287:1265-1269.

44. Chromy, B.A., et al. 2003. Self-assembly of Abeta(142) into globular neurotoxins. Biochemistry. 11:12749-12760.

45. LeVine, H., III. 2004. Alzheimer's [beta]-peptide oligomer formation at physiologic concentrations. Anal. Biochem. 335:81-90. 